\title{
ADULT INTESTINAL MALROTATION: CASE REPORT
}

\author{
Má rotação intestinal em adulto: relato de caso
}

\author{
Ubirajara Rutilio M. e F. de ARAÚJO, Imad Izat EL TAWIL
}

From Ônix Medical Center, Curitiba,PR, Brasil.

\author{
Correspondência: \\ Ubirajara Araújo, \\ e-mail: biraraujo@bol.com.br
}

Conflict of interest: none

Financial source: none

Submited: 10/08/2009

Accepted for publication: 25/01/2011

\section{INTRODUCTION}

I ntestinal malrotation is a congenital anomaly caused by incomplete rotation or no rotation of the intestine in superior mesenteric artery axis during embryogenesis ${ }^{2}$. Typically presents in early life, but sometimes it can present later causing difficulty and error in diagnosis 9 . It is known that $64 \%$ of cases become clinically evident during the first months of life and $82 \%$ in the first year ${ }^{9}$ although some authors claim that up to $90 \%$ of cases are diagnosed by completing one year' ${ }^{9}$. The true incidence of this malformation is unknown, with estimates ranging from 1:200 to 1:6000 live births ${ }^{4}$. In adults, the incidence is $0.2 \%{ }^{5}$, and approximately $15 \%$ of all patients with confirmed diagnosis remain asymptomatic throughout life $^{6}$. For these reasons, the diagnosis of this disease is part of day-to-day of pediatric surgery, but this diagnosis is often overlooked in cases of abdominal pain in adults ${ }^{1}$.

\section{CASE REPORT}

Woman of 21 years, telephone operator, was admitted to hospital with signs of severe abdominal pain during three days and progressively worsened over the past 24 hours. The pain was diffuse type, coliky, but more intense in the epigastric region and radiating to the back. She showed significant worsening after meals, when had also nausea and vomiting. She denied any other complaints.

There was no comorbidity, but reported similar episode three years earlier, however improved spontaneously with bowel rest and anti-spasmodic.

On physical examination she was in good general condition, slightly dehydrated, normal colored, and afebrile with stable vital signs ( $\mathrm{BP}=110 \times 60 \mathrm{mmHg}$ and
$\mathrm{HR}=80 \mathrm{bpm}$ ). The abdomen was flat, flaccid, slightly painful on palpation of the epigastrium, but no signs of peritoneal irritation.

Were requested laboratory tests (CBC, electrolytes, liver and pancreatic function) that were within normal limits, as well as abdominal ultrasonography that showed only total bowel distension. Analgesics and proton pump blocker were done and an upper endoscopy showed only a small hiatal hernia. On the second day of admission the clinical picture remained unchanged, but more food intolerance A tomography of the abdomen concluded intestinal malrotation along the axis of the superior mesenteric artery, with signs of sub-occlusion of the upper gastrointestinal tract.

It was done a laparotomy. Intraoperatively the small intestine was in the right side of the abdomen and the colon on the left. In addition, the proximal jejunum was in an ischemic volvulus of $720^{\circ}$ about on the axis of the superior mesenteric vessels; the right colon was behind these vessels but with their vascular arcade above them. For the correction of the anomaly an enterotomy was done in proximal jejunum, about 10 $\mathrm{cm}$ from the duodenojejunal angle, and liberation of the volvo which progressive improvement of intestinal ischemia, allowing an enteroanastomosis. The right colon was compressing the superior mesenteric vessels, causing vascular engorgement. Followed the ligation of the pedicle of the middle colic artery at its origin and right colectomy with laterolatera ileotransversostomy.

The patient recovered well and was discharged on the $5^{\text {th }}$ day after surgery and remained asymptomatic until the last follow-up, two years after the operation.

\section{DISCUSSION}

According to some authors, the vast majority of cases of intestinal malrotation in adults are asymptomatic ${ }^{4,5,9}$, among those who develop symptoms, changes may occur as an acute or chronic form. The acute course with abdominal pain and vomiting without distention (obstruction is high) can lead to intestinal ischemia and necrosis with peritonitis ${ }^{1}$. This can be caused by intestinal obstruction by the volvulus or internal hernia, with or without intestinal ischemia ${ }^{2,5}$. In general, with good history its possible to find a history of intermittent symptoms, but mild ${ }^{1}$. 


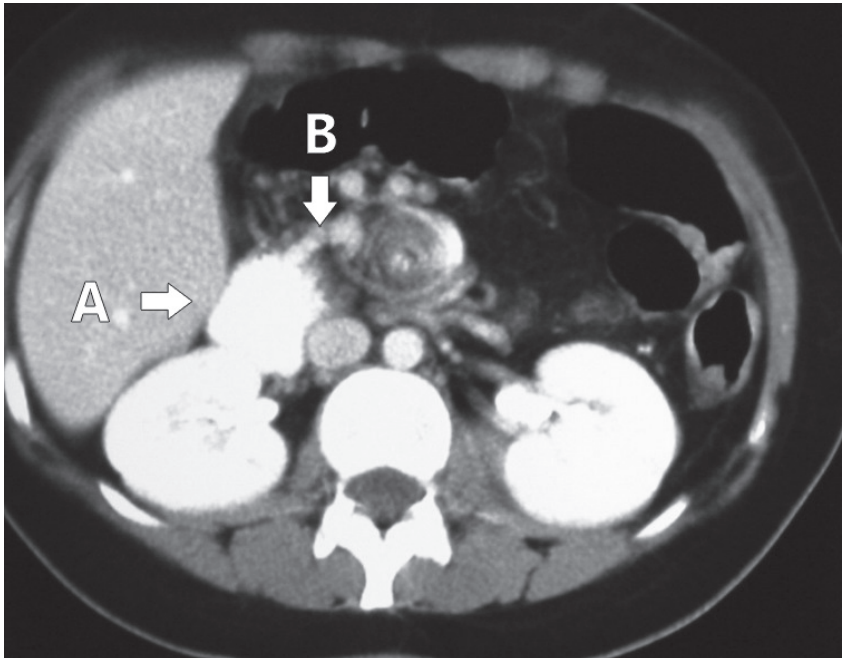

FIGURE 1 - Aspects of abdominal CT: a) dilation and thickening of the duodenal wall and $b$ ) duodenojejunal transition shifted to the right of midline

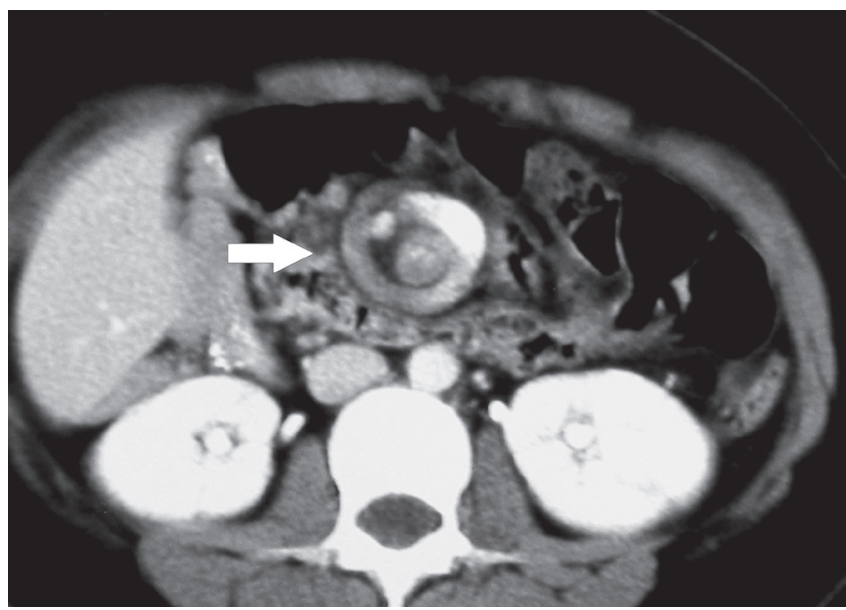

FIGURE 2 - Intestinal rotation $720^{\circ}$ about the axis of the superior mesenteric vessels

In this case, evolution was acute caused by volvulus of the jejunum with subsequent intestinal obstruction. In cases of chronic evolution, symptoms are nonspecific, such as crampy abdominal pain and intermittent and recurrent vomiting ${ }^{1,6}$. The cause of this anomaly is the incorrect setting of the small intestine, causing narrowing of the pedicle of superior mesenteric vessels, which predisposes the intestine volvulus ${ }^{5,6}$. Moreover, they form bundles of peritoneum to try to fix compensatorily the caecum (which is located in the upper right quadrant) in the abdominal wall; these bundles crossing the malpositioned duodenum can cause obstruction or create spaces for the formation of internal hernias ${ }^{5,6}$. These vague symptoms make the diagnosis unclear, slowing it, and often the patient is treated by several doctors, who attribute the complaints to intestinal motility problems or psychiatric disorders $2,3,4,5,6$.

This case had the diagnosis clarified by contrast tomography of the abdomen. This exam has been increasingly requested and shows characteristic changes of anatomical parameters, such as: inverse relationship between the superior mesenteric vessels (vein is located to the left of the artery) ${ }^{1,4,10}$, absence or hypoplasia of the uncinate process of the pancreas ${ }^{4,10}$ and mislocation of the bowel ${ }^{4}$. However, the diagnosis could have been done by radiographic contrast study of upper gastrointestinal tract, which according to many authors, is the gold standard for diagnosis of intestinal malrotation ${ }^{1,4}$, with accuracy exceeding $80 \%{ }^{2,6}$ and in some studies reaching $100 \% 5$. The most important finding to note is the duodenojejunal junction, which does not cross the midline and is located to the right of the spine ${ }^{1,4,6}$. Besides these, there are other diagnostic examinations such as ultrasound of the abdomen (also evaluating the inverse relationship between the mesenteric vessels) $)^{3,6,9,10}$, angiography ${ }^{3,6}$ and laparoscopy ${ }^{7}$. In acute cases, however, the diagnosis in most cases is still done by laparotomy ${ }^{3}$.

Finally, with regard to treatment, there is no standardization for adults, and will depend on the intraoperative findings and the type of associated malformation ${ }^{4}$. But in general must be made the maneuvers called Ladd procedure, which is the release of existing adhesions, mobilization of the duodenum and right colon, release and extension of the pedicle of superior mesenteric vessels and conducting prophylactic appendectomy $y^{1,2,4,5,8}$. There is no consensus in doing cecopexia ${ }^{4}$. Currently, it has been suggested to perform Ladd's procedure by laparoscopy, that has good results and low morbidity 4,7,8. According to some studies, laparoscopic compared to the conventional procedure, is significantly shorter in postoperative hospital staying and decreased need for postoperative analgesia ${ }^{5}$. With respect to asymptomatic cases, there is no consensus on the approach to be used ${ }^{4}$. There are authors who claim that asymptomatic patient should not operate ${ }^{1}$, but there are others, however, who claim that surgical treatment should be instituted to prevent complications caused by volvulus or internal hernia ${ }^{5,6}$.

\section{CONCLUSION}

Intestinal malrotation in adults has difficult diagnosis because inicially the surgeons do not think on it in medical assistance. So is wise to consider its possibility when a prolonged undiagnosed abdominal pain is refered.

\section{REFERENCES}

1. Dietz DW, Walsh RM, Grudfest-Broniatowski S, Lavery IC, et al. Intestinal malrotation: a rare but important cause of bowel obstruction in adults. Dis Colon Rectum 2002, 45(10):1381-6.

2. Gamblin TC, Stephens Jr RE, Jhonson RK, Rothwell M. Adult malrotation: a case report and review of the literature. Curr Surg 2003, 60(5):517-20. 
3. Jayathillake A, Shields MA. Malrotation with volvus: a rare cause of acute bowel obstruction in na adult. ANZ J Surg 2005, 75(9):831-3.

4. Krapfer SA, Rappold JF. Intestinal malrotation - not just the pedriatric surgeon's problem. J Am Coll Surg 2004, 199(4):628-35.

5. Matzke GM, Dozois EJ, Larson DW, Moir CR. Surgical management of intestinal malrotation in adults: comparative results for open and laparoscopic Ladd procedures. Surg Endosc 2005, 19(10):1416-9.

6. Maxson RT, Franklin PA, Wagner CW. Malrotation in the older child; surgical management, treatment, and outcome. Am Surg 1995, 61(2):135-8.

7. Mazziotti MV, Strasberg SM, Langer JC. Intestinal rotation abnormalities without volvulus: the role of laparoscopy. J Am Coll Surg 1997, 185(2):172-6.

8. Seymour NE, Andersen DK . Laparoscopic treatment of intestinal malrotation in adults. JSLS 2005, 9(3):298-301

9. Vukie Z. Presentation of intestinal malrotation syndromes in older children and adults: report of three cases. Croat Med J 1998, 39(4):455-7.

10. Zissin R, Rathaus V, Oscadchy A, Kots $E$, et al. Intestinal malrotation as na incidental finding on CT in adults. Abdom Imaging 1999, 24(6):550-5. 